selves. The action of acids upon aryl-oxyxanthenols has been the stbject of numerous investigations. ${ }^{1}$ It has been generally reported that they gave rise to colored "oxonium" salts of the constitution (III). We have made a systematic study of this reaction with respect to the oxyxanthenols as an introduction to the study of the constitution of fluoran, fluorescein, hydrochinonphthalein, etc.

Our results, as far as they have gone, differ materially from those reported in the literature. We find: (I) that methoxy- and hydroxyxanthenols, except as specified below under (3), yield colorless carbinol chlorides, just like the other xanthenols studied; (2) that these carbinol chlorides tautomerize with extreme readiness to the quinocarbonium salts in the presence of excess of acid, forming acid chlorides; (3) that when one of the two phenyl nuclei in the xanthone ring contains a hydroxy group in the para position to the carbinol carbon atom, then the normal carbinol halides, even in the absence of excess of acid, tautomerize spontaneously to the colored quinoid state, similarly to the benzo- $r$-pyronoles. ${ }^{2}$

In this preliminary paper we shall merely give the results as regards the composition of these colored salts. The method of preparation, the properties of the colorless carbinol halides, their various reactions, the double salts, etc., will be given in a later paper.

\begin{tabular}{|c|c|c|c|}
\hline \multirow[b]{2}{*}{ The Carbinol-hydrochloride of } & \multicolumn{2}{|c|}{ Total chlorine. } & \multirow{3}{*}{ Carbinol chloride. } \\
\hline & Found. & Calculated $\mathrm{Cl}_{2}$. & \\
\hline Phenyl-2-methoxyxanthenol & 19.56 & 19.76 & \\
\hline Phenyl-3-methoxyxanthenol & 19.38 & 19.76 & colorless \\
\hline Phenyl-4-methoxyxanthenol & 19.53 & 19.76 & colorless \\
\hline$p$-Anisylxanthenol $\ldots \ldots \ldots$ & $19 \cdot 7^{8}$ & 19.76 & colorless \\
\hline Phenyl-2-hydroxyxanthenol ${ }^{4}$ & 20.35 & 20.56 & colorless \\
\hline Phenyl-3-hydroxyxanthenol & 20.32 & $20.5^{6}$ & yellow, insoluble \\
\hline Phenyl-4-hydroxyxanthenol ${ }^{3}$ & $20.4 \mathrm{I}$ & $20.5^{6}$ & colorless \\
\hline$p$-Hydroxy-phenylxanthenol & $20.3^{8}$ & 20.56 & colorless \\
\hline
\end{tabular}

ANN ARBOR, MICH.

\title{
THE ADSORPTION OF SOME SUBSTANCES BY STARCHES.
}

By HOYES LLOYD.

Received May 20, 1911.

Wolff and Fernbach ${ }^{8}$ found that some properties of starch and starch

'Baeyer, Ann., 372, 97 (1910); Decker and Fellenberg, Ann., 356, 312 (1907); Kropp and Decker, Ber., 42, 578 (I909); Kehrmann and Dengler, Ber., 4I, 3440 (1908); 42, 870 (rgog); Kehrmann, Ann., 372, 287 (rgro); Pope and Howard, J. Chem. Soc., 97, 1023 (I910); 99, 545 (1911).

2 Gomberg and Cone, Ann., 370, 196 (1909).

${ }^{3}$ The nomenclature in this table is that given by Richter, Lexikon (3rd Ed.); Baeyer ( $A n n ., 372,98)$ suggests a different system.

"Kropp and Decker (Ber., 442, 578) describe a red chloride. Also Baeyer (Ann., $372,104)$.

${ }^{5}$ Baeyer (loc. cit.) describes a red chloride.

${ }^{\circ}$ Compt. rend., 140, 1403-6. 
paste were altered if ordinary lime-containing water instead of distilled water was used for washing the starch. The temperature at which a suspension of starch granules became a translucent liquid was different for the two cases. The viscosity of the paste produced and its acid properties were also different. They say that differences in the properties of the natural starches may be similarly explained.

The properties which Wolff and Fernbach found altered after limecontaining water had reacted with the starch probably indicate that lime had been adsorbed by the starch.

Demoussy ${ }^{1}$ treated rice starch, which had been demineralized by dilute hydrochloric acid and washed till it gave no further traces of chlorine, with a number of acid salts, bases, and neutral salts. He found some of the substance removed from solution in every case and concludes that "starch possesses an acid energy comparable to that of carbonic acid and it should act on salts with a facility corresponding to the weakness of their acids." The reactions with bases are also attributed to the acid properties of starch.

It seems more reasonable to consider the loss of a neutral salt such as potassium chloride from solution to be caused by adsorption of that salt.

Apparently, no complete investigation of these adsorption phenomena has yet been made. This paper gives the results of experiments to test the adsorption of three substances, hydrochloric acid, sodium hydroxide and sodium chloride by cassava, arrowroot, potato, rice, and maize starch granules.

\section{Starches Used.}

Cassava.-A mixture of three samples sent to the Canadian National Exhibition from the West Indies. Two were from St. Vincent and one from St. Lucia.

Arrowroot.--A mixture of eleven samples from different estates in the West Indies-chiefiy from St. Vincent.

Potato.-Supplied by the Edwardsburg Starch Co.

Rice.-This sample was slightly alkaline, as would be expected from the method of preparation--see Jones. ${ }^{2}$

Maize.-The "unchemicalled" brand, ${ }^{3}$ manufactured by the Edwardsburg Starch Co. It contained no alkali.

The amount of difference in moisture content of the various starches used was not great enough to cause much variation in results. Moisture determination is entirely arbitrary in the case of starches; hence, the results in the tables are calculated to moist and not to dry starch. The

${ }^{1}$ Compt. rend., 142, 933-5.

"Eng. Patent, I840, No. 8488. Thorpe's "Dictionary of Applied Chemistry."

${ }^{3} \mathrm{~J}$. Soc. Chem. Ind., 29, 527-3I. 
moisture content determined by heating for two hours averaged $100^{\circ}$, at about i 3 per cent.

\section{Method of Operation.}

In every case, 50 grams of starch were taken and put to shake with the liquid, which was of known strength and always of roo cc. volume. Aqueous solutions were used throughout.

The temperature effect seemed to be very slight. At first the shaker was kept in a thermostat at $0^{\circ}$, but this was found to be unnecessary. Practically no difference was observed between points obtained from experiments done at zero and duplicates done at room temperature for maize and for arrowroot starches. Consequently, all experiments were done at room temperature, $20^{\circ}-24^{\circ}$.

When the liquid has been thoroughly shaken up with the starch, the effect of further stirring was negligible. Thirteen hours' stirring gave only a slightly greater adsorption effect than five minutes' stirring for maize, arrowroot, and potato starch. To insure thorough mixing and to break up any lumps of starch, the bottles were each shaken for five minutes. The rapidity of the reaction in reaching equilibrium points decidedly toward the fact that we are studying a case of simple adsorption.

After shaking, the liquid was separated from the starch and titrated. With starches such as arrowroot and potato, where the granules are large, the suspension settled rapidly and the clear liquid was decanted on a $9 \mathrm{~cm}$. filter paper. The adsorption produced by the filter paper could be neglected, as it was small in comparison to that produced by 50 grams starch. In the case of rice starch, which settles very slowly because of the smallness of the granules, an ordinary centrifuge for milk testing was used. The results produced were the same whether the suspension was settled and decanted, or centrifuged, for centrifuging wide-necked stoppered bottles of about $30 \mathrm{cc}$. capacity were found more convenient than the ordinary Babcock bottles.

The indicator used for hydrochloric acid and for sodium hydroxide was methyl orange. This was chosen in order to neglect the carbon dioxide present and also because the organic acids present would have least effect on it. Phenolphthalein gave a greater acidity because of carbon dioxide and organic acids and the end point was not so sharp.

The reversibility of the reaction was tested. For example, A was made up thus: 50 grams starch $+60 \mathrm{cc}$. normal hydrochloric acid; B was made up thus: 50 grams starch $+60 \mathrm{cc}$. normal hydrochloric acid +40 cc. water. Then both were shaken for five minutes. Then $40 \mathrm{cc}$. of water were added to $A$, which made its total composition the same as that of $B$. After shaking again, both $A$ and $B$ were centrifuged and the filtrate was titrated. If solution $A$ was weaker than solution $B$, the 
water had not washed the hydrochloric acid from the starch granules. Solution A was found to be only slightly weaker than solution $B$, so the reaction may be considered resersible for this concentration. It is doubtful whether this reaction is reversible for starch in solutions of hydrochloric acid weaker than 0.05 normal. Attempts were made at washing the starch iree from hydrochloric acid. It is difficult to test the starch for chlorides and so this was abandoned.

In subsequent tables the strength of the equilibrium solution, after the reaction, is given, column I, in normality. The corresponding figures

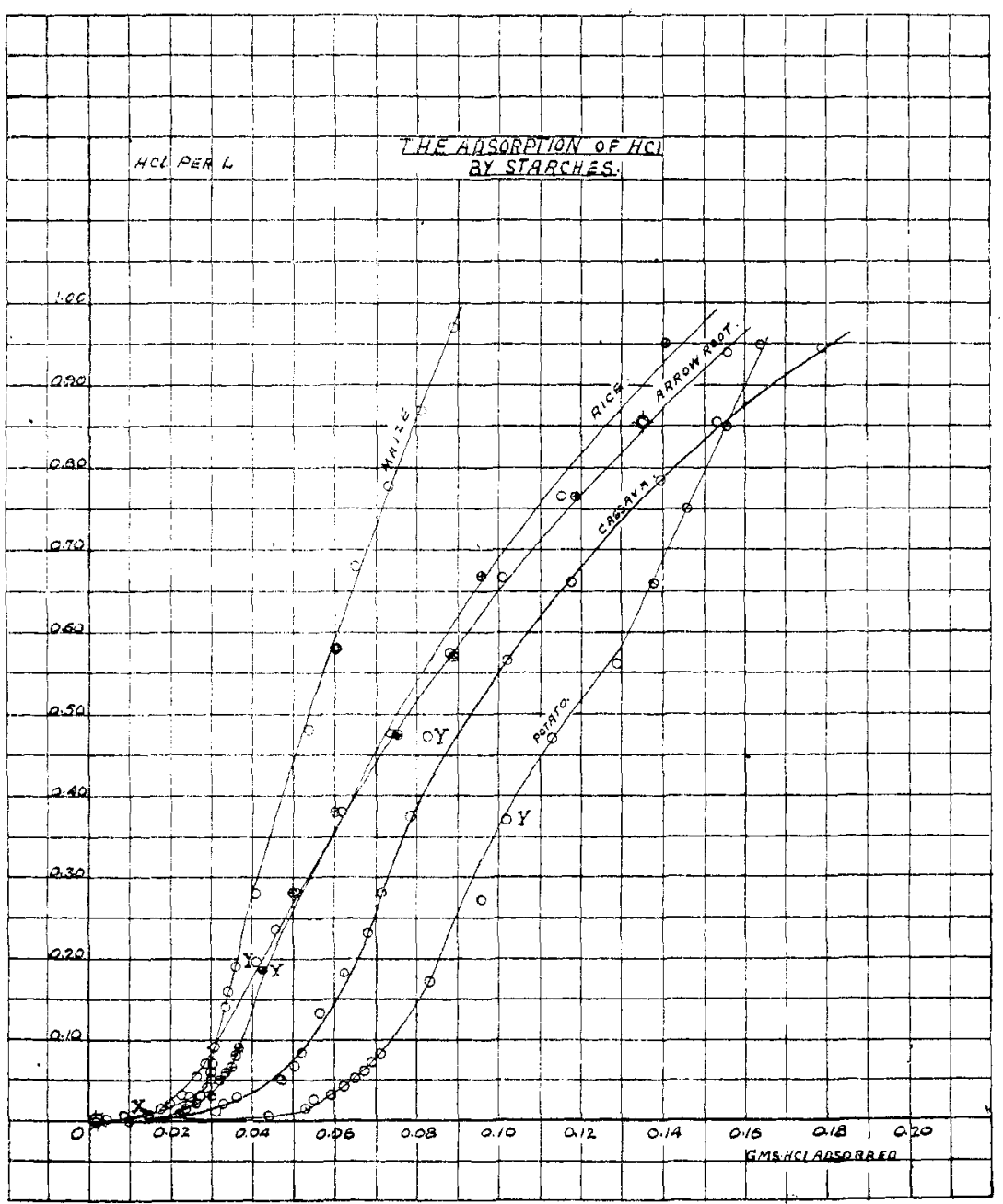

Fig. 1 . 
in column II give, in grams, the weight of hydrogen chloride, sodium hydroxide, or sodium chloride adsorbed by 50 grams starch in equilibrium with this solution. The tables and curves (Fig. I) for hydrochloric acid with starches follow.

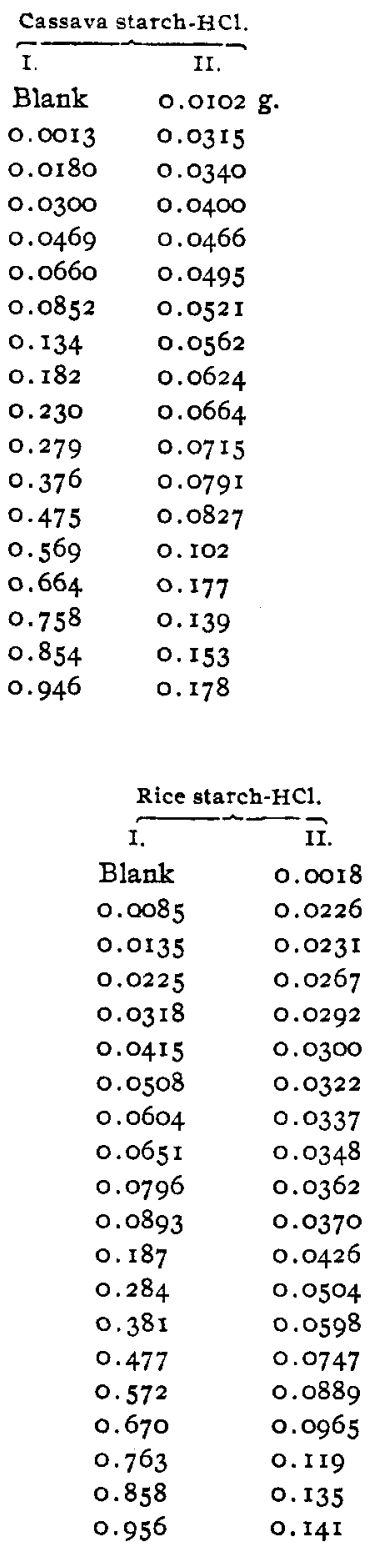

Arrowroot starch-HCl.

I. II.

Blank $0.0016 \mathrm{~g}$.

$0.0072 \quad 0.0098$

0.0147

0.0188

0.0328

0.0236

$0.052 \mathrm{I}$

0.0277

0.0714

0.0294

0.0908

0.0318

0.198

0.0413

0.236

0.0464

0.284

0.38 I

0.0515

0.0624

0.477

o. 0747

$0.087 \mathrm{I}$

o. IOI

o. I I 5

o. I 35

o. 156
Potato starch-HCl.

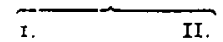

Blank $0.004^{8} \mathrm{~g}$.

$0.0013 \quad 0.0314$

$0.0078 \quad 0.044 I$

$0.0152 \quad 0.0534$

$0.0246 \quad 0.055$ I

$0.0334 \quad 0.0593$

0.04250 .0626

$0.0519 \quad 0.0647$

$0.0612 \quad 0.0670$

$0.0705 \quad 0.0694$

$0.0801 \quad 0.0709$

o. $176 \quad 0.0834$

$0.272 \quad 0.096 \mathrm{r}$

$0.370 \quad 0.102$

$0.466 \quad 0.113$

$0.56 \mathrm{I} \quad 0.128$

$0.658 \quad 0.138$

$0.756 \quad 0.146$

$0.853 \quad 0.155$

$0.950 \quad 0.164$

Màize starch-HCl.

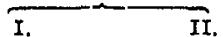

Blank $\quad 0.0007$

$0.0056 \quad 0.0159$

$0.0185 \quad 0.0206$

$0.0231 \quad 0.0246$

$0.0320 \quad 0.028 \mathrm{I}$

$0.0417 \quad 0.0290$

$0.0516 \quad 0.0296$

$0.0614 \quad 0.030 I$

$0.0712 \quad 0.0305$

$0.0910 \quad 0.0306$

$0.140 \quad 0.0339$

$0.159 \quad 0.0346$

o. $189 \quad 0.0363$

$0.287 \quad 0.0406$

$0.483 \quad 0.0537$

$0.58 \mathrm{I} \quad 0.0595$

$0.679 \quad 0.0646$

$0.776 \quad 0.0731$

$0.873 \quad 0.0812$

$0.971 \quad 0.0885$

The equation $a=k c^{p}$ expresses an ordinary adsorption effect where, 


$$
\begin{aligned}
a & =\text { amount adsorbed by } 50 \text { grams starch. } \\
c & =\text { concentration of solution at equilibrium. } \\
k \text { and } p & =\text { constants. }
\end{aligned}
$$

From this equation

$$
\log a=\log k+p \log c
$$

which is the equation of a straight line. Since this is the case, if corresponding values for $\log a$ and $\log c$ were plotted, we should get a straight line as long as the adsorption is following the ordinary law and as long as no other reaction occurs.



Fig. 2. 
Log $a$ and $\log c$ are plotted (Fig. 2) for the various starches with hydrochloric acid. It appears that solutions of hydrochloric acid in which $c$ does not exceed about 0.35 normal are the only ones to obey the above law at all accurately in the case of starches. In Fig. 2 the portion of the curves from $\mathrm{X}$ to $\mathrm{Y}$ appears to follow the law quite closely.

$k$ and $p$ have been calculated and a solution for the cassava curve follows.

Taking two values for $\log a$ and $\log p$,

$$
\begin{array}{cc} 
& -\mathrm{I} .5027=\log k-2.0144 p \\
& -\mathrm{I} . \mathrm{I} 777=\log k-0.6372 p \\
\text { Subtracting } & -0.3250=-1.3772 p \\
& p=0.3250 / \mathrm{I} .377=0.23 .
\end{array}
$$

\begin{tabular}{|c|c|c|}
\hline Starch. & $k$. & b. \\
\hline Cassava.... & 0.093 & 0.23 \\
\hline Arrowroot......... & 0.067 & 0.30 \\
\hline Potato............. & 0.118 & 0.20 \\
\hline Rice. & 0.062 & $0.2 \mathrm{I}$ \\
\hline
\end{tabular}

Substituting $p$ in one of the above equations,

$$
\begin{gathered}
-\mathrm{I} .5027=\log k-(2.9 \mathrm{I} 4 \times 0.236) \\
\log k=-\mathrm{I} .03 \mathrm{I}=\overline{2} .969^{\circ} \\
k=0.093 .
\end{gathered}
$$

A comparison of $k$ and $p$ follows:

In general, the adsorption as shown by the starches in Fig. I increases rapidly to about 0.04 gram where the equilibrium solution is about 0.05 normal. Then the increase of the amount adsorbed becomes not nearly so large for equal increases in strength of the acid. A slight deviation to the right, above the point 0.05 normal, might be due to a penetration effect, following the ordinary surface effect of the adsorption. If this were so, a shortening of the time of stirring should distinguish it from the adsorption effect, which probably takes place immediately; but, as mentioned previously, the time of stirring did not canse a noticeable variation even when the time was altered from one minute to thirteen hours. The amount of hydrochloric acid adsorption seldom exceeds 6 per cent. of the total acid used when normal solution is put on 50 grams of starch.

The hydrolysis produced by normal hydrochloric acid in the cold and in a period of five minutes is entirely negligible. In case of many hours contact between normal hydrochloric acid and starch, some sugars were produced as indicated by Fehling's solution. Oecbsner de Coninck ${ }^{1}$ records the time required to produce substances that reduce Fehling's solution. Using 35-40 cc. water, 3 grams starch, and $2 \mathrm{cc}$. of concentrated

${ }^{1}$ Bull, acad. roy. Belg., 1910, 515-7; C. A., 5, 394 (19ז r). 
hydrochloric acid, he allowed the reaction to proceed at $14^{\circ}$ for three days. Substances that reduced Fehling's solution were found in the liquid after filtration. After six days such substances were found abundantly. Consequently, in these experiments, the amount of hydrolysis is small and so we are dealing witin starch granules. As hydrolysis does not remove the hydrochloric acid from solution but uses up starch, its effect would be to lessen, not to increase the adsorption.

The experimental error is large, owing to variation in the 50-gram samples of starch and also to the fact that the amount adsorbed is found by subtracting the amount of the hydrochloric acid after the reaction. from the amount in the solution used. Titrations of $50 \mathrm{cc}$. were used or $50 \mathrm{cc}$. were diluted to $250 \mathrm{cc}$. and an aliquot part titrated. The experimental error is greater for the stronger solutions because the amount of the adsorption is small in comparison with the amount of hydrochloric acid present. In these curves, a few points falling a long distance from the curve were rejected as erratic.

Now, adsorption is a surface effect and the amount of the adsorption depends on the surface of the particles. The sutface per gram of rice starch is approximately ten times that of potato starch and one would consequently expect greater adsorption effects for rice starch than for the other starches, stich as potato starch, which have larger granules and less surface per gram. Potato starch, however, and arrowroot, less notably, have a concentric series of markings which may materially increase the surface of these starches per gram. The starches are dissimilar enough to expect to find differences in the amounts of chemical adsorbed by equal amounts of various starches under similar conditions.

\section{Surface Per Unit Weight.}

I have worked ont, roughly, the area of surface in 50 grams of three of the starches used. The surface of one granule was calculated, using the arerage size for the particular kind of starch as given by Archbold. ${ }^{1}$ Then the approximate number of granules per gram was estimated by calculating the volume of one granule and using the average specific gravity of starch. The total area for 50 grams is large, as would be expected considering the smallness of the granules.

The equation for the surface of an ellipse was used for potato starch. Where

$$
\begin{gathered}
e=\sqrt{a^{2}-b^{2} / a} \\
a \text { and } b=\text { half axes. } \\
A=2 \pi a b / e\left\{e \sqrt{1-e^{2}}+\sin ^{-1} e\right\}
\end{gathered}
$$

The granules of rice and maize were considered spherical although this is not strictly accurate.

: J. Soc. Chem. Ind., 22, 63-66. 
Starch.

Area of I granule in sq. $\mathrm{mm}$.

Volume of I granwle in cubic nm.

Rice. . . . . . . . 0.0000502

Maize. . . . . . . . . 0.0006408

Potato...... . . 0.002947
0.0000000335
0.000001532
0.00001716

Diameter of $t$ granule in $\mathrm{mm}$.

$0.004 \times 0.004$

$0.0142 \times 0.0142$

$0.040 \times 0.027$
Area of $50 \mathrm{~g}$ in $\mathrm{sq}$. meters.

$49.7 \mathrm{I}$

13.93

$5 \cdot 99$

The Adsorption of Sodium Hydroxide by Starches.

Symons ${ }^{1}$ has investigated the process of swelling of starch granules under the action of heat and alkaline solutions of varying strengths. He shows that most granules are swollen and a suspension is converted

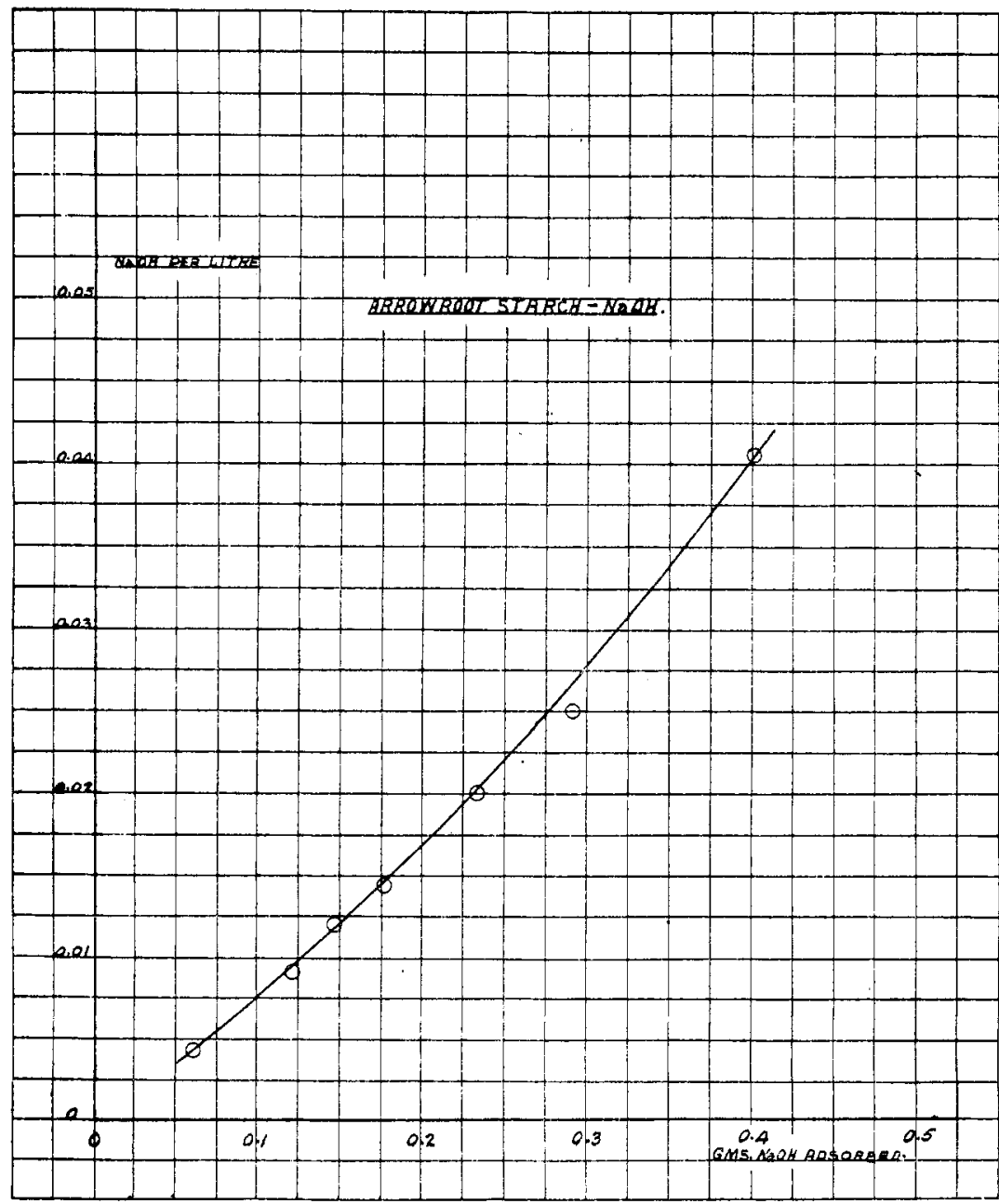

Fig. 3.

1 Thorpe's "Dictionary of Applied Chemistry," pp. I I I-562; Pharm. J.and Trans., $3,13-237$. 
into a paste at a point where the solution contains about one per cent. sodium hydroxide.

Brown and Heron ${ }^{1}$ state that starch, which has been treated with potash and acid in the process of purification, produces a paste of less viscosity than the same starch which has not been so treated. This is a result

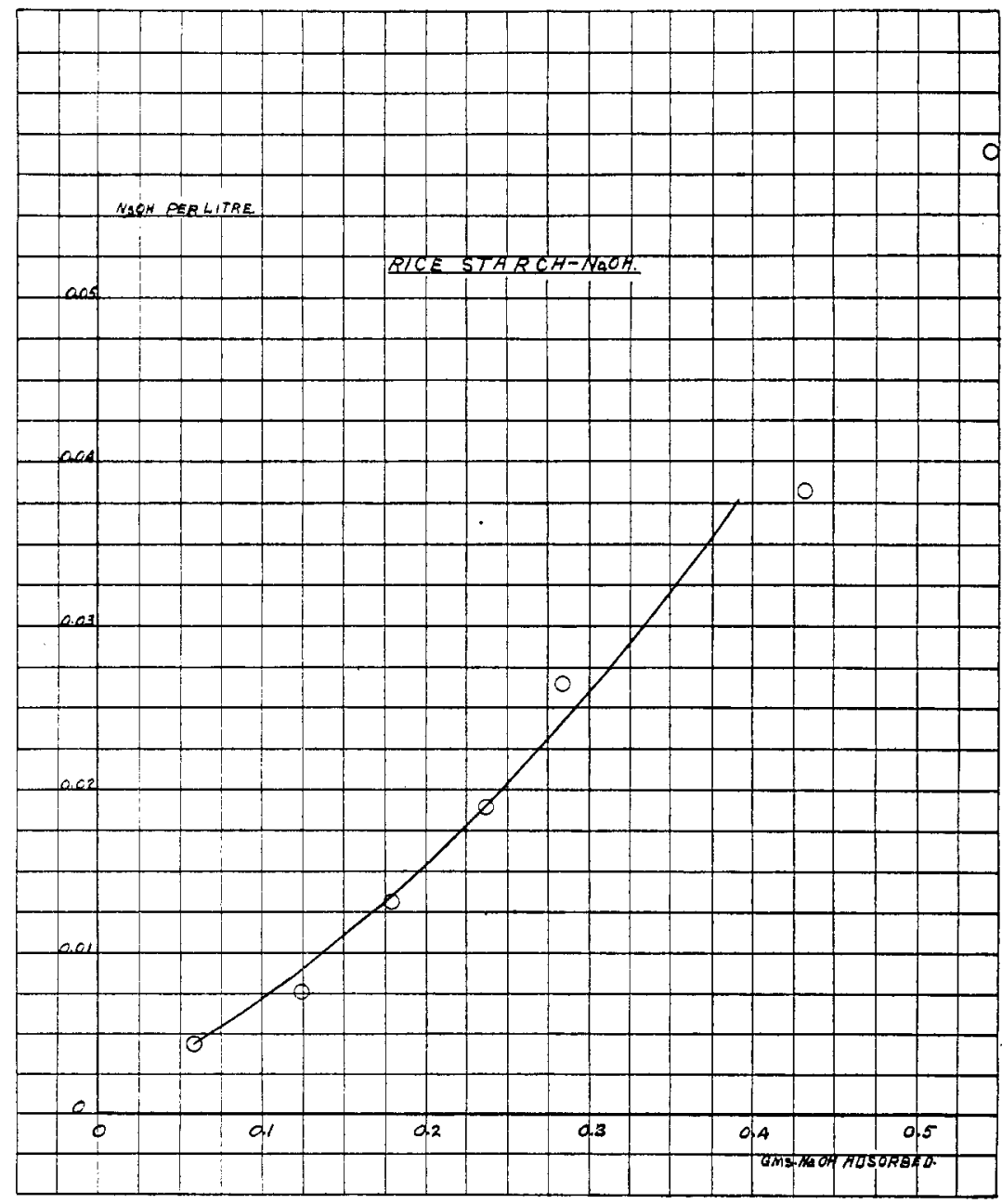

I'ig. 4 .

probably produced by alkalies adsorbed by the starch granules. They found that when a moderately strong solution of potash or soda was added to an opalescent starch paste, the liquid became perfectly transparent.

${ }^{1}$ Am. Chem. J., 35, 596. 
This fact and the fact that the specific rotatory power is considerably lowered, but resumes its original value if the starch be neutralized, they claim to indicate a definit compound of starch with potash and soda.

In these experiments, concentrations greater than 0.2 normal were not used because the starch gelatinizes with stronger solutions. One

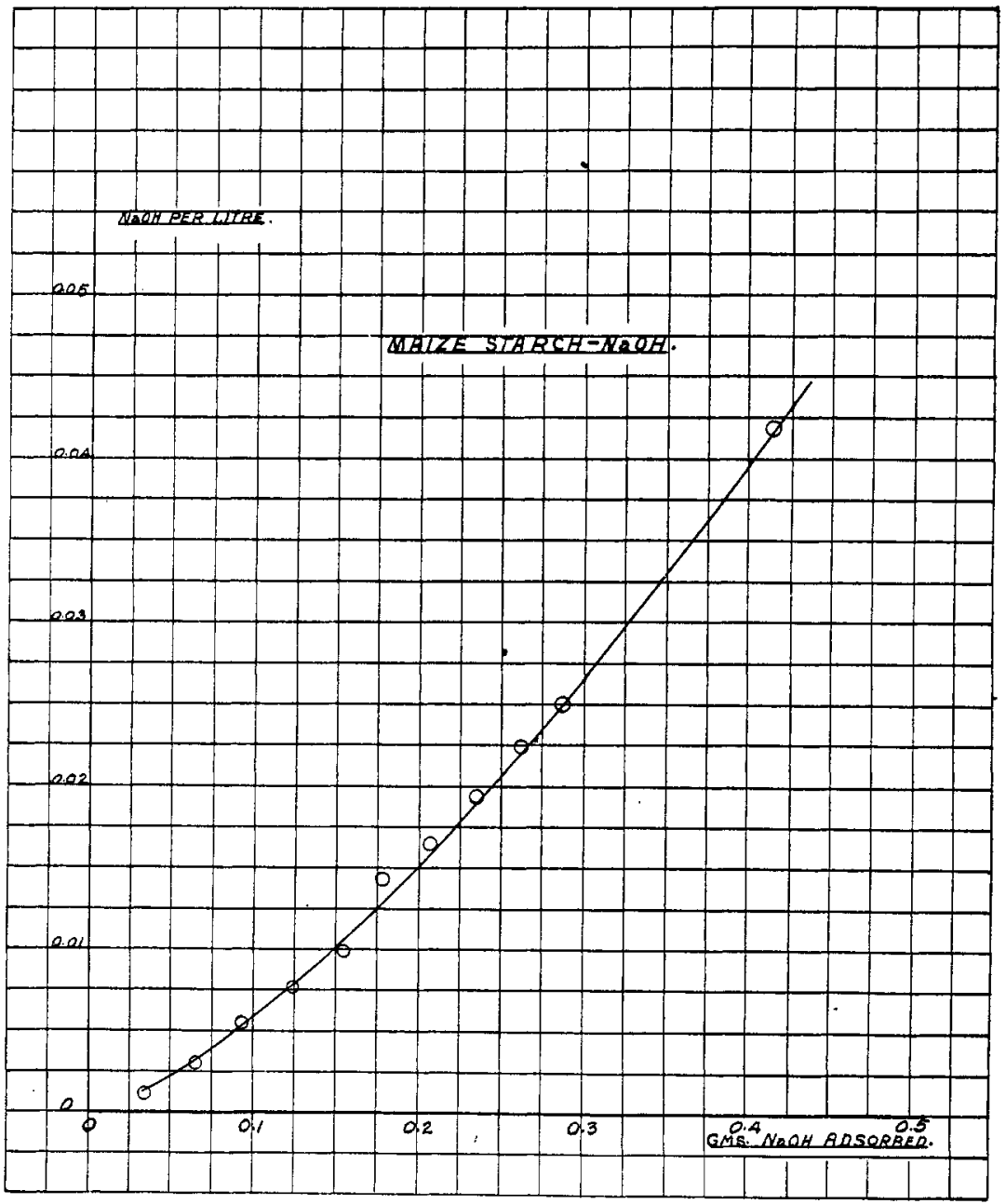

Fig. 5 .

minute stirring gave results which compared with thirteen hours' stirring. The equilibrium is reached quickly and is not followed by a penetration effect.

The tables and curves show the experimental results for arrowroot 
(Fig. 3), rice (Fig. 4), and maize starch (Fig. 5). The verticalax is gives the normality of sodium hydroxide in the filtrate after the reaction. The horizontal axis gives, in grams, the amount of sodium hydroxide adsorbed by 50 grams starch. The amount of the adsorption is large in the case of sodium hydroxide.

\begin{tabular}{cclll} 
Arrowroot starch-NaOH, & & \multicolumn{2}{c}{ Kice starch-NaOH. } \\
\hline I. & II. & & I. & II. \\
0.00438 & 0.0606 & & 0.00438 & 0.0606 \\
$0.01 \mathrm{II}$ & 0.112 & & 0.0077 & 0.125 \\
$0.012 \mathrm{I}$ & 0.146 & & 0.0132 & $0.18 \mathrm{I}$ \\
0.0145 & 0.176 & & 0.0188 & 0.237 \\
0.0200 & 0.232 & & 0.0263 & 0.285 \\
0.0254 & 0.288 & & 0.0382 & 0.433 \\
0.0402 & 0.425 & & 0.0589 & 0.545 \\
0.0563 & 0.556 & & &
\end{tabular}

\begin{tabular}{|c|c|}
\hline \multicolumn{2}{|c|}{ Maize starch.NaOr } \\
\hline I. & II. \\
\hline 0.0010 & 0.0347 \\
\hline 0.0030 & $0.066 \mathrm{I}$ \\
\hline 0.0054 & 0.0955 \\
\hline $0.007^{6}$ & 0.125 \\
\hline 0.0099 & o. I 55 \\
\hline 0.0142 & 0.177 \\
\hline 0.0164 & 0.207 \\
\hline 0.0193 & 0.235 \\
\hline 0.0224 & 0.262 \\
\hline $0.025^{6}$ & 0.288 \\
\hline 0.0427 & $0.4 I 5$ \\
\hline 0.0545 & $0.5^{6} 3$ \\
\hline
\end{tabular}

Maize starch- $\mathrm{NaCl}$

\begin{tabular}{lllc} 
I. & \multicolumn{1}{c}{ II. } & \multicolumn{2}{c}{ Arrowroot starch-NaCl. } \\
Blank & 0.00294 & I. & II. \\
0.0193 & 0.0137 & Blank & 0.00463 \\
0.0364 & 0.0186 & 0.0181 & 0.0098 \\
0.0556 & 0.0218 & 0.0369 & 0.0156 \\
0.0752 & 0.0228 & 0.0557 & 0.0213 \\
0.0947 & 0.0244 & 0.0744 & 0.0276 \\
0.1933 & 0.0269 & 0.0952 & 0.0273 \\
0.287 & 0.0561 & 0.189 & 0.0486 \\
0.386 & 0.0584 & 0.285 & 0.0663 \\
0.479 & 0.0862 & 0.377 & 0.107 \\
0.576 & 0.103 & 0.566 & 0.158 \\
0.665 & 0.159 & 0.660 & 0.191 \\
0.762 & 0.185 & 0.753 & 0.223 \\
0.855 & 0.207 & 0.850 & 0.235 \\
0.949 & 0.235 & &
\end{tabular}

The Adsorption of Sodium Chloride by Starches.

Demoussy ${ }^{1}$ mentions that sodium chloride reacts with starch because of the acid properties of the starch. He does not give the effect of change in concentration on the amount of the adsorption. His experiments were all done with rice starch, which had been previously treated with hydrochloric acid.

The solutions were nade from Kahlbaum's sodium chloride as it contained no magnesium salts. The sodium chloride in the filtrate after reaction with the starch was determined by titrating with o. I $N$ silver nitrate solution, using potassium chromate as indicator. The time of

'Compt. rend., 142, 933-5. 
stirring was five minutes and the temperature $20^{\circ}-24^{\circ}$. The results are given for arrowroot and maize starch (Fig. 6). The vertical axis

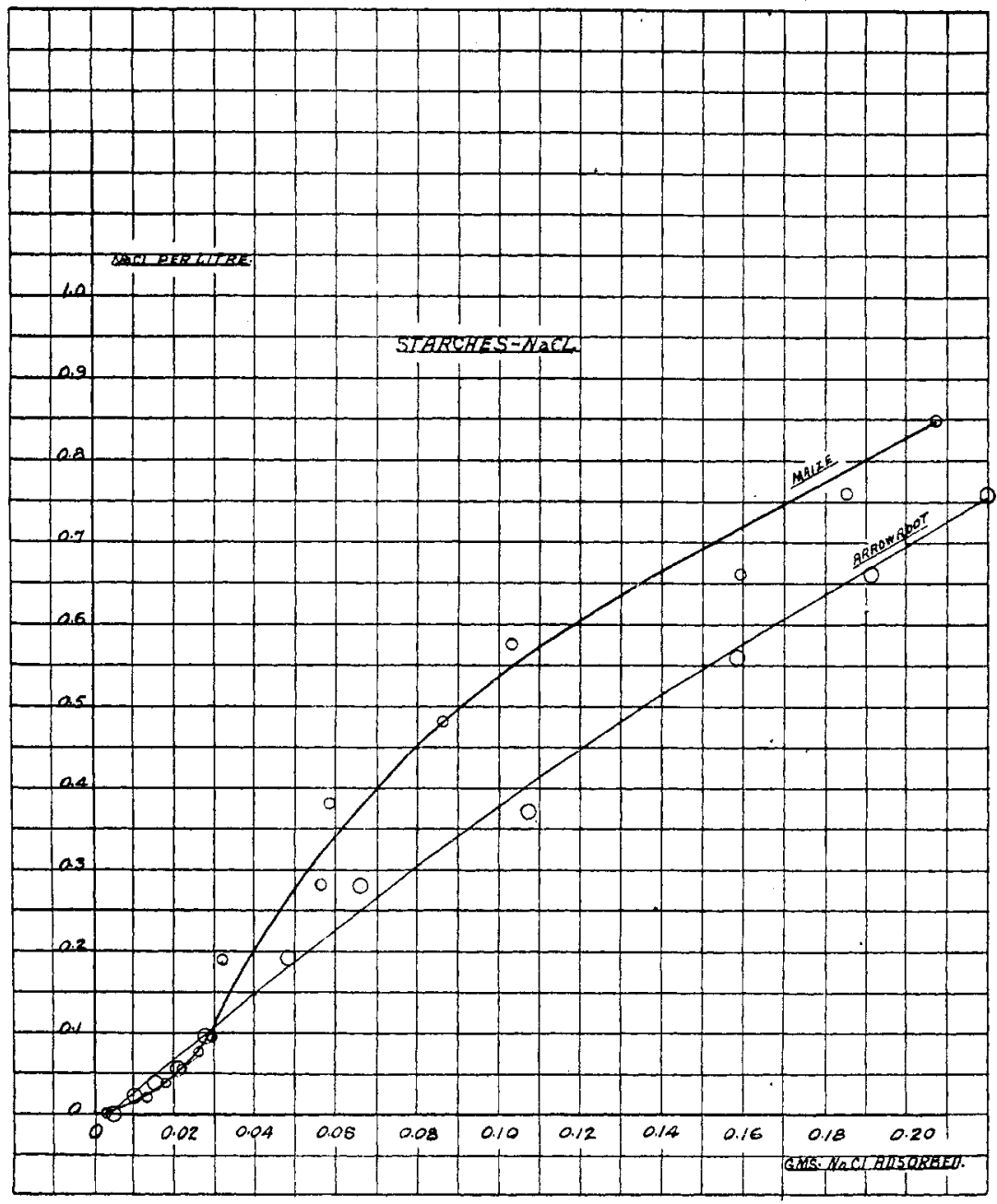

Fig. 6.

gives the normality of sodium chloride after the reaction; the horizontal gives, in grams, the sodium chloride adsorbed by 50 grams starch.

\section{Conclusion.}

I. The adsorption of hydrochloric acid, sodium hydroxide, and sodium chloride, by starch, varies with different starches but not as much as would be expected, considering the great differences in the size of the granules. 
2. The adsorption is not a function of the granule surface per unit weight.

3. The amount of tie adsorption is much greater for sodium hydroxide than for either hydrochloric acid or sodium chloride.

4. In the case of starch-hydrochloric acid, the ordinary adsorption rule is followed for sulutions up to about $0.4 \mathrm{~N}$, except in the case of maize starch.

My thanks are due Proiessor Lang, The Staff in Chemistry, and Dr. W. P. Kanfmann for lind assistance. Some samples vere provided by the Imperial Commission on Agriculture for the West Indies.

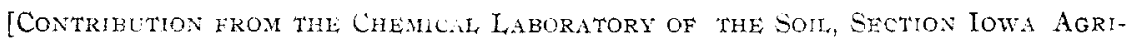
CILTLRAL FXPERIMENT STAJTOX.]

\title{
THE CHEMICAL NATURE OF THE ORGANIC NITROGEN IN THE. SOIL.
}

\author{
BY is I,. Jojisis. \\ Leceived April $\therefore$, $19: 1$
}

The data given in this paper, as well as in a previous one, ${ }^{1}$ show, in agreement with numerous investigations by others, that the annount of ammonia in soils is quite small, ranging from a few thonsands to a few hundreds of one per cent. The proportion of nitrites and nitrates, too, is in the great najority of cases misignificant, being but a few thousands of one per cent. and less, which is true of the soils herein examined. Hence, practically all of the nitrogen occurring in soils is of ats organic nature. This enphatically points to the importance of restarches with a view to finding out the nature of the organic nitrogenous compounds present in the soil. Such knowledge would enable us, in the first place, to better understand the inportant biological processes of ammonification, nitrification and denitrificalion: to comprehend why the nitrogen in one soil is more useful or a ailable than in another; to see whether there are among the nitrogenous conpounds such that are detrimental to plant life; and last but not least, it will enable us to utilize the soil nitrogen to better advantage than is now the case.

While some nitrogenous bodies like urea, for instance, readily furnish ammonia through fermentation, others do not. While such compounds like aspragine, $6 . .$. represent plant food, tyrosine is considered as being hurtful to plant; whereas leucine and similar compounds, in the presence of nitrites, can give rise to the process of denitrification, so much sn that under favorable conditions lencine is thus quantitatively converted in to lencinic acid; other compounds with different chemical structure cannot. Important as is the knowledge of the organic matter in the soil, onr pres-

${ }^{1}$ This Jolruxa, 3I, 396; also Thinical Bul. 4 (rgog), Michigan Agricultural Experiment Station. 